\title{
A comparison of clinically utilized human papillomavirus detection methods in head and neck cancer
}

Nicolas F Schlecht ${ }^{1,2}$, Margaret Brandwein-Gensler ${ }^{3}$, Gerard J Nuovo ${ }^{4}$, Maomi Li ${ }^{5}$, Anne Dunne ${ }^{6}$, Nicole Kawachi ${ }^{7}$, Richard V Smith ${ }^{7,8}$, Robert D Burk ${ }^{1,6}$ and Michael B Prystowsky ${ }^{7}$

${ }^{1}$ Department of Epidemiology and Population Health, Albert Einstein College of Medicine, Bronx, NY, USA;

${ }^{2}$ Department of Medicine (Oncology), Albert Einstein College of Medicine, Bronx, NY, USA; ${ }^{3}$ Department of Pathology, University of Alabama Birmingham, Birmingham, AL, USA; ${ }^{4}$ Department of Pathology, Ohio State University College of Medicine, Columbus, OH, USA; ${ }^{5}$ Esoterix Genetic Laboratories, LLC, Successor to Genzyme Genetics* , New York, NY, USA; ${ }^{6}$ Departments of Pediatrics (Genetics), Microbiology and Immunology, Obstetrics and Gynecology and Women's Health, Albert Einstein College of Medicine, Bronx, NY, USA $;{ }^{7}$ Department of Pathology, Albert Einstein College of Medicine, Bronx, NY, USA and ${ }^{8}$ Department of Otorhinolaryngology-Head and Neck Surgery, Montefiore Medical Center, Bronx, NY, USA

Detection of human papillomavirus (HPV) in head and neck cancer has therapeutic implications. In situ hybridization and immunohistochemistry for $\mathrm{p} 16$ are used by surgical pathologists. We compared the sensitivity and specificity of three popular commercial tests for HPV detection in head and neck squamous cell carcinomas with a 'gold standard' HPV PCR assay. A total of $\mathbf{1 1 0}$ prospectively collected, formalin-fixed tumor specimens were compiled onto tissue microarrays and tested for HPV DNA by in situ hybridization with two probe sets, a biotinylated probe for high-risk (HR) HPV types 16/18 (Dako, CA, USA) and a probe cocktail for 16/18, plus 10 additional HR types (Ventana, AZ, USA). The p16 $16^{\mathrm{INK}}$ expression was also assessed using a Pharmingen immunohistochemistry antibody (BD Biosciences, CA, USA). Tissue microarrays were stained and scored at expert laboratories. HPV DNA was detected by MY09/ 11-PCR, using Gold AmpliTaq and dot-blot hybridization on matched-fresh frozen specimens in a research laboratory. HPV 16 E6 and E7-RNA expression was also measured using RT-PCR. Test performance was assessed by a receiver operating characteristic analysis. HR-HPV DNA types 16, 18 and 35 were detected by MY-PCR in $28 \%$ of tumors, with the majority (97\%) testing positive for type 16 . Compared with MY-PCR, the sensitivity and specificity for HR-HPV DNA detection with Dako in situ hybridization was 21\% (95\% confidence interval (Cl): 7-42) and $100 \%$ (95\% Cl: 93-100), respectively. Corresponding test results by Ventana in situ hybridization were $59 \%$ (95\% Cl: 39-78) and 58\% (95\% Cl: 45-71), respectively. The p16 immunohistochemistry performed better overall than Dako $(P=0.042)$ and Ventana $(P=0.055)$, with a sensitivity of $52 \%(95 \% \mathrm{Cl}: 32-71)$ and specificity of $93 \%(95 \% \mathrm{Cl}: 84-98)$. Compared with a gold standard HPV-PCR assay, HPV detection by in situ hybridization was less accurate for head and neck squamous cell carcinoma on tissue microarrays than p16 immunohistochemistry. Further testing is warranted before these assays should be recommended for clinical HPV detection.

Modern Pathology (2011) 24, 1295-1305; doi:10.1038/modpathol.2011.91; published online 13 May 2011

Keywords: diagnostic methods; head and neck neoplasms; human papillomavirus; immunohistochemistry; in situ hybridization; PCR; p16

Correspondence: Dr NF Schlecht, PhD, Department of Epidemiology and Population Health, Albert Einstein College of Medicine, 1300 Morris Park Avenue, Bronx, NY, 10641, USA.

E-mail: nicolas.schlecht@einstein.yu.edu

${ }^{*}$ Genzyme Genetics and its logo are trademarks of Genzyme Corporation and used by Esoterix Genetic Laboratories, LLC, a wholly owned subsidiary of LabCorp, under license. Esoterix Genetic Laboratories and LabCorp are operated independently from Genzyme Corporation.

Received 13 December 2010; revised 24 March 2011; accepted 24 March 2011; published online 13 May 2011
Head and neck cancer remains an important problem in the United States, with over 30000 cases and 7800 deaths occurring annually. ${ }^{1}$ Detection of human papillomavirus (HPV) in head and neck squamous cell carcinoma has therapeutic implications. HPV, particularly type 16 , is detected in a third of head and neck squamous cell carcinoma and is associated with improved survival and response to therapy. ${ }^{2,3}$ As a result, the most recent American Joint Committee on Cancer (AJCC) staging criteria 
recommends reporting HPV status especially for oropharyngeal cancer, where detection rates are highest. ${ }^{4} \mathrm{~A}$ number of commercial assays, including DNA, RNA and protein-based tests, are available. However, detection rates vary considerably across studies, and there is no consensus which, if any, is adequate for diagnostic evaluation of HPV in head and neck squamous cell carcinoma.

In situ hybridization has been used for HPV DNA detection in both primary and metastatic tumors by pathologists for a number of years, although its effectiveness as a clinical diagnostic tool in head and neck squamous cell carcinoma has not been tested extensively. ${ }^{5}$ Alternatively, p16 $6^{\mathrm{INK} 4 \mathrm{a}}$ expression measured by immunohistochemistry has also been suggested as a surrogate for transcriptionally active HPV16. ${ }^{6-10}$ The current gold standard used in HPV research includes PCR-based assays designed to detect either HPV DNA or RNA. Among the commercial tests readily available to pathologists, both p16 immunohistochemistry and HPV in situ hybridization can be performed on formalin-fixed and paraffin-embedded tissue, and allow for direct visualization of target protein or nucleic acid in tumor cells. However, independent comparison of these multiple clinically-utilized HPV detection methods is limited for head and neck squamous cell carcinoma. ${ }^{11-13}$ Critical evaluation of HPV detection assays in head and neck squamous cell carcinoma is growing in importance as HPV-associated tumors may require different therapeutic approaches. We compared the sensitivity and specificity of three popular commercial tests for HPV detection (two direct and one indirect) in prospectively collected head and neck squamous cell carcinoma primary tumors with gold standard HPV PCR assays.

\section{Materials and methods}

\section{Collection of Clinical Samples and Patient Data}

Matched-fresh frozen and formalin-fixed and paraffin-embedded primary head and neck squamous cell carcinoma tumor samples were collected prospectively from 109 patients. Subsequent first and second head and neck squamous cell carcinoma primaries were collected from one patient. All patients with primary head and neck squamous cell carcinoma treated at Montefiore Medical Center were approached for participation at time of diagnosis. Tumor samples were collected following histological confirmation and snap-frozen in liquid nitrogen in the same pathology laboratory within minutes of surgical resection or biopsy. Additional biopsies were collected before therapy for research purposes and processed in the same fashion. Patient demographics and tobacco smoking and alcohol consumption histories were also collected by medical interview before treatment. The study protocol was approved by the Institutional Review Boards at
Montefiore Medical Center and Albert Einstein College of Medicine, and all patients provided written informed consent. Histological confirmation of tumors was performed by a single pathologist (MBG), assessing tumor grade and staging on the basis of the AJCC classification.

\section{Tissue Microarrays}

Tissue microarray blocks were constructed from formalin-fixed and paraffin-embedded tissue, using a semi-automatic tissue arrayer (Chemicon) and $1.0 \mathrm{~mm}$ cores, which contained three or more cores per sample from the areas with maximal numbers of tumor cells. Microarray sections were deparaffinized, rehydrated and washed in TBS (Sigma; tris buffer T6664) before staining.

\section{HPV In-situ Hybridization}

Tissue microarray sections were stained for HPV DNA by in situ hybridization, with two different probe sets: INFORM HPV-III Fam16(B) probe cocktail (Ventana, AZ, USA) for 12 high-risk (HR) types (HPV16, 18, 31, 33, 35, 39, 45, 51, 52, 56, 58 and 66) and an HPV 16/18 biotinylated DNA probe set (Y1412), with GenPoint tyramide single amplification system (Dako, CA, USA). Tissue microarray sections were stained and scored independently at two expert laboratories blinded to tumor site and results by the other assays. Examples of HPV DNA positive head and neck squamous cell carcinoma detected by Dako and Ventana are shown in Figure 1.

\section{p16 Immunohistochemistry}

Deparaffinized tissue microarray sections were incubated with a Pharmingen immunohistochemistry monoclonal mouse antibody (BD Biosciences, CA, USA) at 1:50 dilution for $30 \mathrm{~min}$ at room temperature, followed by addition of secondary Dako EnVision biotinylated horseradish peroxidase-conjugated mouse antibody (K4001) for $30 \mathrm{~min}$ at room temperature without dilution, then stained with ultra-marque diaminobenzidine reagent for 2-4 min (Dako; K3468) and finally counterstained with hematoxylin (Harris formula; Surgipath, IL, USA). An example of a p16 immunohistochemistry positive staining head and neck squamous cell carcinoma is shown in Figure 2. All tissue microarray slides, plus controls, were stained in one assay. Positive and negative controls consisted of uterine cervix with severe dysplasia, with and without primary antibody, respectively. Immunohistochemical expression for p16 was quantified according to cellular location (nuclear and cytoplasmic), staining intensity (on a scale of 0-3) according to the strongest signal that comprised at least $10 \%$ of each 

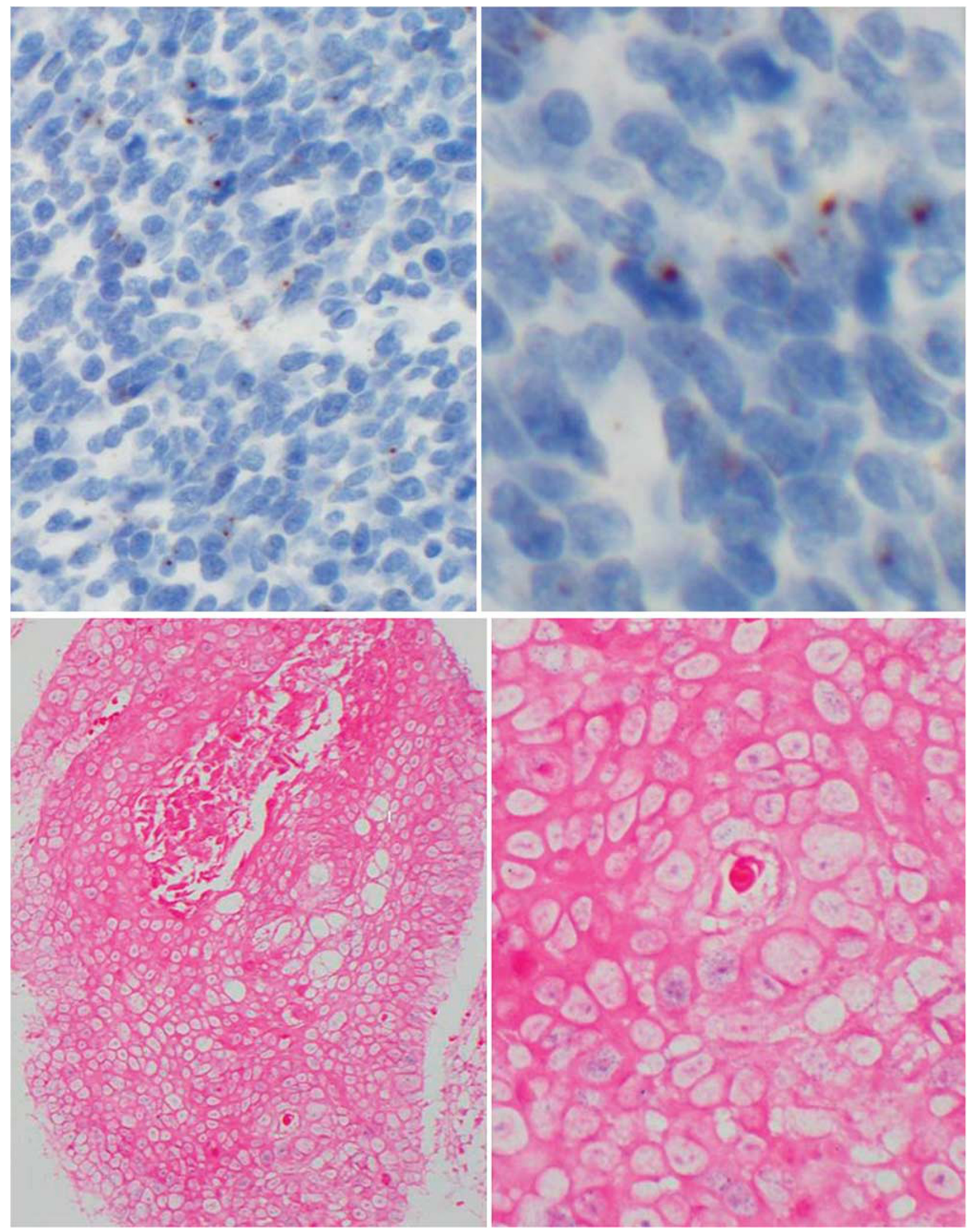

Figure 1 Top: Dako in situ hybridization positive case showing brown intranuclear signals, this tumor was HPV16 positive by PCR (left panel: low power, right panel: high power). Bottom: Ventana in situ hybridization positive case showing blue/purple intranuclear signals, this tumor was HPV16 positive by PCR (left panel: low power, right panel: high power). 


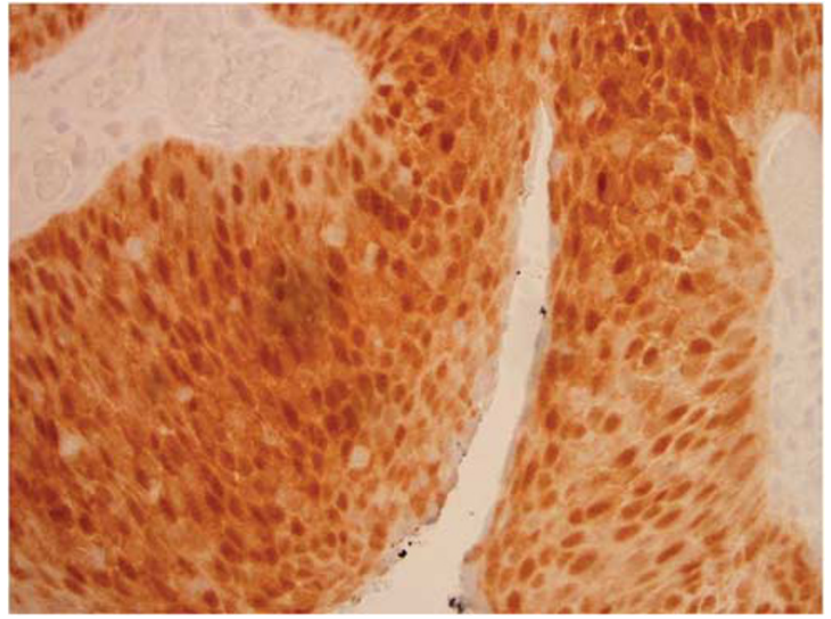

Figure 2 The p16 immunohistochemistry positive case showing strong nuclear and cytoplasmic staining, with a distribution of $\geq 75 \%$ (low power); this tumor was HPV16 positive by PCR.

core and distribution $(0,25,50$ or $75 \%)$ of antibody stains in tumor cells.

\section{HPV DNA Detection and Genotyping by PCR}

Genomic DNA was isolated from 106 matched-fresh frozen tissues using the DNEasyTissue Kit (Qiagen, CA, USA). HPV DNA was detected by a MY09/ MY11/HMB01-PCR system using Gold AmpliTaq DNA polymerase that amplifies a $450 \mathrm{bp}$ segment in the L1-sequence of HPV as described. ${ }^{14}$ HPV genotyping was performed on all samples by dotblot hybridization, using biotinylated oligonucleotide probes for 40 HPV types, including those tested for, by both in situ hybridization assays. To verify the specificity of the hybridizations, a complete set of controls were included on all membranes. The integrity of specimen DNA was verified by amplification of a cellular gene ( $\beta$-globin) ${ }^{14}$ In addition, control samples 'spiked' with DNA from a cervical carcinoma cell -line, with known quantity of HPV16 genomes, were included in every assay. DNA viral load was also assessed by MY-PCR dot-blot intensity on a scale of $1-5 .{ }^{14}$

\section{HPV16 E6 and E7 mRNA Detection}

RNA samples, isolated from 86 matched-fresh frozen tumors were tested for HPV16 E6 and E7 transcripts, found in over $80 \%$ of HPV positive head and neck squamous cell carcinomas. Total RNA was extracted and purified by a standardized protocol using TRIzol (Invitrogen, Carlsbad, CA, USA). RNA was not available for 25 head and neck squamous cell carcinoma. Samples were subjected to a multiplex RT-PCR assay, using a series of oligonucleotide primers that span the 204-525 nucleotide E6 and E7 regions of the HPV16 genome, following a standardized protocol. ${ }^{15,16}$ The primers were designed to detect the full-length and spliced E6 mRNA trans- cripts. E7-encoding mRNA primers were designed on the basis of HPV16 sequence NC_001526 (complete genome), using the web-based Primer3 design program (available at http://frodo.wi.mit.edu/primer3). The performance of the primers was confirmed using control RNA samples from HPV positive and negative head and neck squamous cell carcinoma and cervical carcinoma cell lines: FaDu, SCC-25, CAL27, SCC-15, UM-SCC-1, UM-SCC-47, UPCI-SCC-090, SiHa and CaSki. In addition, glyceraldehyde-3-phosphate dehydrogenase (GAPDH) primers were included in each assay to verify the integrity of the specimen mRNA (based on sequence AY340484:Homo sapiens GAPDH gene, complete cds).

\section{Statistical Analyses}

Stained tissue microarray sections were scored at independent expert laboratories; Ventana probes were used at Department of Pathology Laboratory, Ohio State University College of Medicine, OH, USA (GN) and Dako probes at Genzyme Genetics, NY, USA (ML). Positive nuclear staining was ascertained over multiple core replicates from the same primary tumor biopsy or resection specimen. A third pathologist (MBG), blinded to HPV PCR and in situ hybridization results and tumor site, read the tissue microarrays for p16 expression in the cytoplasm and nucleus. Mean staining was then calculated across multiple core replicates from the same primary tumor biopsy or resection. The p16 positivity was defined by a mean intensity cut-off of $\geq 2$ and diffuse $(\geq 75 \%)$ staining distribution in either the nuclei or cytoplasm, derived by sensitivity analysis and corresponding to published criteria. ${ }^{22}$ Histological assessment of keratinization was also completed for 66 tumors, measured on a four-point scale from high $(\geq 75 \%)$ to low $(<25 \%)$.

Test performance was assessed by a receiver, operating characteristic analysis against the HPV PCR results as the gold standard. Areas under the receiver operating characteristic curves and 95\% confidence intervals (CI) were estimated to assess differences in overall test performances. ${ }^{17}$ In situ hybridization and immunohistochemistry results were compared with HR-HPV DNA genotyping results by MY-PCR, and HPV16 E6/E7 RNA expression by RTPCR. Concordance between PCR and in situ hybridization test results was assessed by Kappa statistic and Spearman correlation. Differences in keratinization across tumors were assessed using $\chi^{2}$-tests.

\section{List of Commercial Assays}

Semi-automatic tissue arrayer: Chemicon (now part of Millipore). Millipore, 290 Concord Road, Billerica, MA 01821, USA.

INFORM HPV-III Fam16(B) probe set: Ventana Medical Systems, 1910 E. Innovation Park Drive, Tucson, AZ 85755, USA. 
Dako HPV16/18 biotinylated probe (Y1412) with GenPoint tyramide signal amplification system: Dako North America, 6392 Via Real Carpinteria, CA 93013, USA.

Pharmingen p16 immunohistochemistry monoclonal mouse antibody: BD Biosciences, Pharmingen, 10975 Torreyana Road, San Diego, CA 92121, USA.

\section{Results}

A total of 110 prospectively collected primary head and neck squamous cell carcinoma tumor specimens were tested for HPV DNA by PCR, HPV in situ hybridization and p16 immunohistochemistry. Specimens were collected before initiation of chemotherapy or radiation by biopsy $(N=36)$ or surgical resection ( $N=74$, including 21 laser resection cases). Head and neck squamous cell carcinoma tumor sites represented include oropharynx $(N=30)$, hypopharynx $(N=8)$, larynx $(N=32)$, lip/ oral cavity $(N=38)$ and nasopharynx $(N=2)$. The majority of head and neck squamous cell carcinoma were stage IV $(N=66,60 \%)$, followed by stage III $(N=19,17 \%)$, II $(N=14,13 \%)$ and I $(N=11,10 \%)$. The majority of patients were male $(N=76,70 \%)$, with a median age at head and neck squamous cell carcinoma diagnosis between 62 and 63 years. Non-
Hispanic White $(N=43,39 \%)$, followed by Hispanic White $(N=28,26 \%)$ and non-Hispanic AfricanAmerican $(N=26,24 \%)$ patients represented the three largest race and ethnic groups. A history of tobacco smoking and alcohol drinking was also reported by the majority of patients $(N=94,86 \%)$, of which less than half continued to smoke $(N=41$, $37 \%)$ and drink $(N=21,19 \%)$ at time of diagnosis.

HR-HPV DNA was detected by MY-PCR in $28 \%$ $(N=30 / 106)$ of tumors. HPV16 was found in all, but one of the HR-HPV positive cases. Table 1 shows the performance of each of the HPV testing methods (in situ hybridization and immunohistochemistry) relative to HPV MY-PCR and RT-PCR. Compared with MY-PCR, the sensitivity and specificity of HRHPV DNA detection by in situ hybridization using Ventana probes was 59 and $58 \%$, respectively. Corresponding test results for HR-HPV DNA detection by Dako were 21 and $100 \%$. The results for the Dako in situ hybridization test were unchanged when compared with HPV16/18 detection only, as the single case positive for a HR-HPV type other than 16/18 was not discernable by Dako in situ hybridization due to insufficient tumor tissue or core dropout on the corresponding tissue microarray section (see Supplemental Material).

Overall performance of each test in detecting HRHPV DNA (reflecting combined sensitivity and

Table 1 Performance of HPV testing methods relative to PCR in head and neck squamous cell carcinoma

\begin{tabular}{|c|c|c|c|c|c|c|}
\hline & \multirow{2}{*}{$\begin{array}{c}\text { No. of } H P V+ \\
\text { by test }(\%)\end{array}$} & \multirow[t]{2}{*}{ No. tested } & \multicolumn{4}{|c|}{ Gold standard: high-risk HPV DNA by MY-PCR } \\
\hline & & & $\begin{array}{l}\text { Sensitivity } \\
(95 \% \text { CI })\end{array}$ & $\begin{array}{c}\text { Specificity } \\
(95 \% \text { CI })\end{array}$ & $\begin{array}{l}\text { Area under the } \\
\text { curve }(95 \% C I)^{\mathrm{a}}\end{array}$ & $\mathrm{P}$-value ${ }^{\mathrm{b}}$ \\
\hline \multicolumn{7}{|l|}{ All sites combined } \\
\hline p16 Immunohistochemistry & $19(20 \%)$ & 97 & $52 \%(32-71)$ & $93 \%(84-98)$ & $0.72(0.62-0.82)$ & (Reference) \\
\hline In situ hybridization (Ventana) & $41(47 \%)$ & 87 & $59 \%(39-78)$ & $58 \%(45-71)$ & $0.59(0.47-0.70)$ & 0.055 \\
\hline In situ hybridization (Dako) & $5(6.6 \%)$ & 76 & $21 \%(7-42)$ & $100 \%(93-100)$ & $0.60(0.52-0.69)$ & 0.042 \\
\hline \multicolumn{7}{|l|}{ Oropharynx only } \\
\hline p16 Immunohistochemistry & $10(37 \%)$ & 27 & $69 \%(39-91)$ & $93 \%(66-100)$ & $0.81(0.66-0.96)$ & (Reference) \\
\hline In situ hybridization (Ventana) & $16(70 \%)$ & 23 & $67 \%(35-90)$ & $27 \%(6-61)$ & $0.47(0.27-0.67)$ & 0.000 \\
\hline \multirow[t]{3}{*}{ In situ hybridization (Dako) } & $4(21 \%)$ & 19 & $40 \%(12-74)$ & $100 \%(66-100)$ & $0.70(0.54-0.86)$ & 0.046 \\
\hline & & & \multicolumn{4}{|c|}{ Gold standard: HPV16 RNA by RT-PCR } \\
\hline & $\begin{array}{c}\text { No. of } H P V+ \\
\text { by test (\%) }\end{array}$ & No. tested & $\begin{array}{l}\text { Sensitivity } \\
(95 \% \text { CI })\end{array}$ & $\begin{array}{l}\text { Specificity } \\
\text { (95\% CI) }\end{array}$ & $\begin{array}{l}\text { Area under the } \\
\text { curve }(95 \% C I)^{a}\end{array}$ & $\mathrm{P}$-value ${ }^{b}$ \\
\hline \multicolumn{7}{|l|}{ All sites combined } \\
\hline p16 Immunohistochemistry & $18(23 \%)$ & 80 & $56 \%(35-76)$ & $93 \%(82-98)$ & $0.74(0.64-0.85)$ & (Reference) \\
\hline In situ hybridization (Ventana) & $35(48 \%)$ & 73 & $67 \%(45-84)$ & $61 \%(46-75)$ & $0.64(0.52-0.76)$ & 0.600 \\
\hline In situ hybridization (Dako) & $4(6 \%)$ & 67 & $18 \%(5-40)$ & $100 \%(92-100)$ & $0.59(0.51-0.67)$ & 0.045 \\
\hline \multicolumn{7}{|l|}{ Oropharynx only } \\
\hline p16 Immunohistochemistry & $9(43 \%)$ & 21 & $90(56-100)$ & $100 \%(72-100)$ & $0.95(0.85-1.00)$ & (Reference) \\
\hline In situ hybridization (Ventana) & $13(68 \%)$ & 19 & $67 \%(30-93)$ & $30 \%(7-65)$ & $0.48(0.26-0.71)$ & 0.007 \\
\hline In situ hybridization (Dako) & $3(19 \%)$ & 16 & $38 \%(9-76)$ & $100 \%(63-100)$ & $0.69(0.51-0.87)$ & 0.034 \\
\hline
\end{tabular}

Abbreviations: CI, confidence interval; HPV, human papillomavirus.

${ }^{a}$ Receiver operating characteristic area under the curve.

${ }^{\mathrm{b}} P$-value for comparison of receiver operating characteristic curves for in situ hybridization assays to p16 immunohistochemistry assuming independence. 
specificity) was assessed by comparing the areas under the receiver operating characteristic curves. An area under the curve of 0.5 or less indicates a diagnostic test that cannot distinguish HPV positive from HPV negative cases. Compared with p16 immunohistochemistry (area under the curve $=0.72$ ), test performance by Ventana in situ hybridization (area under the curve $=0.59$ ) and Dako in situ hybridization (area under the curve $=0.60)$ were statistically worse $(P=0.055$ and $P=0.042$, respectively). Sensitivity and specificity of p16 immunohistochemistry for detection of HR-HPV (with an intensity cut-off of $\geq 2$ and diffuse $(\geq 75 \%$ ) staining) was 52 and $93 \%$, respectively. However, test performance for p16 immunohistochemistry decreased if a lower intensity $(<2)$ or distribution $(<75 \%)$ cut-point was used, due to a proportionally larger change (loss) in specificity relative to sensitivity (gain). Agreement between Ventana and Dako probes was also poor $(\kappa=0.14$, 95\% CI: 0.02-0.26), although the difference in overall performance between the two in situ hybridization tests for detecting HR-HPV DNA was not significant $(P=0.415)$. Test performance improved only slightly when formalin-fixed and paraffinembedded and fresh frozen specimens were both procured by surgical resection (p16 immunohistochemistry area under the curve $=0.75,95 \% \mathrm{CI}$ : 0.63-0.86; Ventana in situ hybridization area under the curve $=0.64,95 \%$ CI: 0.52-0.76; and Dako area under the curve $=0.62$, 95\% CI: 0.51-0.72), although the differences were not significant.

Looking at HPV DNA prevalence by anatomic site, HR-HPV DNA detection by MY-PCR was highest amongst oropharyngeal tumors $(N=14,47 \%)$, followed by hypopharyngeal $(N=3,43 \%)$, laryngeal $(N=8,26 \%)$ and oral cavity $(N=4,11 \%)$ sites. HPV positive oropharyngeal tumors also tended to have higher viral levels measured by dot blot intensity $($ mean $=4.2 \pm 1.2$ and median $=5$, on a scale of $1-5)$, compared with laryngeal (mean $=2.8 \pm 0.7$, median $=3$ ) or oral cavity tumors (mean $=3 \pm 0.8$, median=3). HR-HPV DNA detection sensitivity in oropharyngeal tumors only was higher for all tests, although overall test performance was only somewhat improved for p16 immunohistochemistry (area under the curve $=0.81,95 \%$ CI: 0.66-0.96) and Dako in situ hybridization (area under the curve $=0.70$, 95\% CI: 0.54-0.86), but not for Ventana in situ hybridization (area under the curve $=0.47,95 \% \mathrm{CI}$ : 0.27-0.67) (Table 1).

Test performance was also assessed against HPV E6/E7 RNA expression as measured by RT-PCR. HPV E6/E7 oncogene expression in cancer is associated with integration of the HPV genome into the host genome and overexpression of p16 following E7-mediated inactivation of pRb. ${ }^{18} \mathrm{HPV} 16$ E6/E7 RNA expression was detected by RT-PCR in 31\% of tumors tested $(N=27 / 86)$. As observed with HPV DNA detection by MY-PCR, p16 immunohistochemistry (area under the curve $=0.74$ ) performed sig- nificantly better than Dako in situ hybridization (area under the curve $=0.59, P=0.045$ ), and somewhat better than Ventana in situ hybridization (area under the curve $=0.64, P=0.600$ ). Overlap in HPV16 E6 and E7 expression was high (97\%, $\kappa=0.92$, 95\% CI 0.83-1.0), and little to no difference in performance was observed when HPV16 E7 and E6 RNA expression were assessed separately. Although test performance compared with HPV16 E6/E7 RNA by RT-PCR was little changed for in situ hybridization (using either probe set) when restricting to oropharyngeal SCC cases, p16 immunohistochemistry was significantly higher (area under the curve $=0.95$, 95\% CI: 0.85-1.00) (Table 1).

We were also able to assess whether p16 expression by immunohistochemistry correlated with tumor histology within the head and neck. Degree of keratinization across head and neck tumors was low (median between 50 and $<25 \%$ ) and was significantly less in oropharyngeal and laryngeal tumors, compared with oral cavity, with 74, 52 and $21 \%$ of tumors showing $<25 \%$ keratinization, respectively, reflecting a mostly basaloid morphology among oropharyngeal tumors. We observed an inverse correlation between p16 staining intensity and degree of keratinization $(\rho=-0.215, P=0.102)$ overall and among oropharyngeal tumors in particular $(\rho=-0.490, P=0.054)$. The p16 immunohistochemistry continued to perform better than Ventana $(P=0.002)$ or Dako $(P=0.121)$ in situ hybridization, compared with HR-HPV DNA detection by MY-PCR when restricted to non-keratinizing tumors.

Contrasting HPV16 DNA and RNA results $(N=82)$, there was good agreement between HPV16 E6/E7 RT-PCR and HPV16-specific MY-PCR results (84\%, $\kappa=0.62$, 95\% CI: $0.44-0.81$ ). Although most cases were HPV16 positive by both MY-PCR (that amplifies a target in L1) and E6/E7 RT-PCR, five $(6 \%)$ did not show HPV16 E6 and E7 oncogene expression and eight $(10 \%)$ had undetectable levels of HPV16 DNA. Three additional cases, negative by MY-PCR, tested positive for either E6 or E7 RNA, but not both-these were considered RT-PCR negative.

We also assessed the potential for using p16 immunohistochemistry in a triage scenario to detect head and neck squamous cell carcinoma tumors expressing HPV oncogenes (ie, E6/E7 RNA by RTPCR), where HR-HPV DNA testing by either in situ hybridization or MY-PCR is used to further discriminate cases, using p16 expression with a cut-off for p16 of $1+$ intensity and $\geq 10 \%$ distribution as positive. Setting this low threshold for p16 resulted in a detection rate of $94 \%$ and sensitivity and specificity of 96 and $7 \%$, respectively (area under the curve $=0.52$, 95\% CI: 0.46-0.57). We saw little to no improvement in detection performance when combining tests with p16 immunohistochemistry as described with MY-PCR (area under the curve $=0.76$, 95\% CI: 0.66-0.87), in situ hybridization by Ventana (area under the curve $=0.66,95 \%$ 


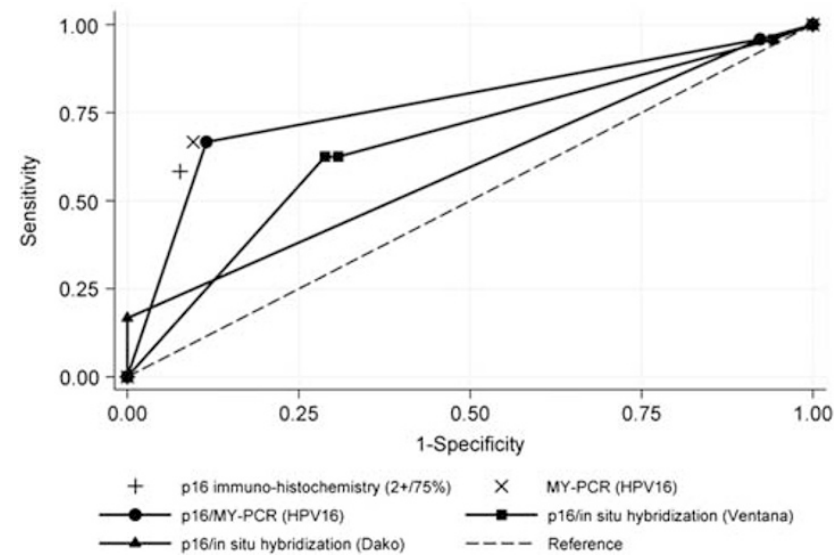

Figure 3 Receiver operating characteristic curves for head and neck squamous cell carcinomas by combined p16 immunohistochemistry and HPV DNA testing by in situ hybridization using the INFORM HPV-III Fam16(B) (Ventana) and HPV16/18 biotinylated GenPoint (Dako) probes or by MY-PCR, compared with HPV16 E6/E7 RT-PCR. Non-parametric receiver operating characteristic curves shown for combined test results with p16 immunohistochemistry (assuming a $1+/ \geq 10 \%$ cut-off) stratified by HPV Ventana in situ hybridization (-ם-), Dako in situ hybridization (- $\mathbf{\Delta}-)$ or MY-PCR (-) results. Solitary markers shown reflect single test performance statistics for p16 IHC (assuming a $2+/ \geq 75 \%$ cut-off) $(+)$ and MY-PCR for HPV16 $(\times)$ alone. The dashed line indicates a reference test threshold with area under the receiver operating characteristic curve of 0.5 .

CI: $0.55-0.78$ ) or in situ hybridization by Dako (area under the curve $=0.58$, 95\% CI: 0.51-0.65; Figure 3). These results were little changed when restricting to oropharyngeal SCC cases only (not shown).

There was little agreement between the in situ hybridization and immunohistochemistry test results among cases discordant by PCR. Among HPV16 RNA + /DNA - cases $(N=8)$, half were positive by Ventana in situ hybridization, two were positive by p16 immunohistochemistry at the $2+/ \geq 75 \%$ cut-off and none were positive by Dako in situ hybridization, with no overlap between in situ hybridization and immunohistochemistry positives. Four of the remaining p16 cases stained with $\geq 2$ intensity, but with distributions of $\leq 50 \%(N=1)$ and $\leq 25 \%(N=3)$. Among HPV16 RNA-/DNA + cases $(N=5)$, only one each were found positive by Ventana in situ hybridization and p16 immunohistochemistry (at the $2+/ \geq 75 \%$ cutoff), whereas the remaining four p16 cases also stained with $\geq 2$ intensity, but with distributions of $\leq 50 \%(N=2)$ and $\leq 25 \%(N=2)$. The majority of discordant cases $(N=9 / 13)$ originated outside the oropharynx, including the anterior tongue, floor of mouth, retromolar trigone, alveolar ridge, posterior pharyngeal wall and supraglottis. Test performance increased only slightly when restricting cases to tumors concordant for HPV16 DNA and RNA detection by PCR $(N=68)$ : p16 immunohistochemistry area under the curve $=0.84$ (95\% CI: 0.730.96), Ventana in situ hybridization area under the curve $=0.66(95 \%$ CI: $0.52-0.79)$ and Dako area under the curve $=0.63$ (95\% CI: 0.52-0.75).

\section{Discussion}

Despite improvements over the last decade, 5-year survival rates for head and neck squamous cell carcinoma remain at $50 \%$. Growing evidence indicates that, for a subset of head and neck squamous cell carcinoma, the presence of HPV in tumors constitutes a positive prognostic marker of disease. ${ }^{2,19}$ Detection of HPV in head and neck squamous cell carcinoma therefore has obvious therapeutic implications in treatment management ${ }^{4}$ or targeted intervention, ${ }^{20,21}$ and pathologists will have a central role in the identification and triaging of HPV positive head and neck squamous cell carcinoma cases.

We therefore asked, if detection of HPV in head and neck squamous cell carcinoma is important for treatment decisions, which test is most appropriate for accurate detection of HPV? To investigate this, we independently tested three commercial, clinically utilized HPV detection methods (two direct, one indirect) available to anatomic pathologists. This study represents one of the first systematic comparisons of these common HPV detection methods for head and neck squamous cell carcinoma. ${ }^{22}$

HR-HPV prevalence rates in our study varied considerably by detection method and anatomic site, with the highest estimates obtained for oropharyngeal tumors by in situ hybridization, using the Ventana Fam16 probe cocktail. Other studies, such as Agoston et al's, ${ }^{13}$ have reported higher detection rates in oropharyngeal tumors using in situ hybridization with the Ventana Fam16 probe cocktail, and PCR using L1 and E7 DNA primers, but did not provide analogous estimates of test performance as comparisons were made to combined test results assuming no false positives. Sensitivity and specificity estimates for p16 immunohistochemistry using a Dako antibody (G175-405) relative to PCR were both higher $(100 \%)$ and lower $(35 \%)$, respectively, compared with our results with the Pharmingen antibody. Singhi and Westra ${ }^{12}$ used the Dako in situ hybridization platform as the 'gold standard' for comparing p16 immunohistochemistry in oropharyngeal cancers, which we show in this study to be least sensitive. Nonetheless, they reported higher ( $>80 \%$ ) prevalence rates for HPV in a population of patients referred for suspicion of HPV; either to determine eligibility for clinical trials, guide clinical diagnosis or requested by concerned patients and their partners.

The advantage of immunohistochemistry for p16 is that, it is readily available in surgical pathology laboratories and relatively simple to evaluate. Typical p16 positive cases usually show diffuse, strong nuclear and cytoplasmic expression. Indeter- 
minate staining patterns not reaching this cut-off value were relatively common in this study. This may in part be a sampling issue related to our use of tissue microarray cores; it is possible that the frequency of limited distribution of staining is less common when tissue sections are examined. This may also have been a factor for the in situ hybridization staining, especially where positive cells are few and focal, as found with the Dako GenPoint probe.

Notably, there is a lack of published consensus as to what cut-off should be used for the determination of p16 positivity, with some groups setting low cutoff points. ${ }^{7,23-27}$ When restricting to the oropharynx, the head and neck site most likely to be associated with HPV, we detected no HPV16 RNA negative tumors with p16 staining, with a distribution of $\geq 75 \%$, whereas only one HPV16 RNA positive case had p16 staining, with a distribution of $<75 \%$. Lewis et $a l^{28}$ also reported p16 staining with a distribution of $\leq 75 \%$ in oropharyngeal SCC tumor cells in only $7 \%$ of p16 'positive' cases.

In contrast, in situ hybridization probe signals can be more difficult to interpret than immunohistochemistry staining, and non-specific signals are a well recognized problem. ${ }^{29}$ Moreover, a distinction is made between intranuclear vs paranuclear or cytoplasmic staining on in situ hybridization. This specification allows for the identification of clini- cally relevant HPV infections in cancer cells. However, this can also lead to ambiguous staining results in situ and lower sensitivity, compared with PCR. For example, whereas specific localization within the nucleus was needed for interpretation of Dako staining as positive, purple nuclear staining by Ventana probes was more frequently interpreted as positive in our experience. An example of an HPV PCR negative head and neck squamous cell carcinoma with discordant HPV in situ hybridization results by Ventana (more sensitive test) and Dako (more specific test) is shown in Figure 4.

Results can also differ by pathologist. Comparison with blinded reading of the staining results by our laboratory pathologist (MBG) revealed higher sensitivity (33 vs 21\%), but lower specificity (94 vs 100\%) with Dako in situ hybridization than the expert evaluation, and higher specificity ( 86 vs 58\%) and equal sensitivity with Ventana in situ hybridization (59\%), compared with MY-PCR. In response, automated imaging methods have recently been incorporated into commercial labs. However, even after removing individual subjectivity in interpretation, technical variability with hybridization can remain an obstacle. In contrast, PCR-based HPV tests have been in use in research for over 20 years and have the added advantage of providing type-specific information. ${ }^{30-32}$ PCR methods targeting the HPV L1 region are also used in clinical HPV testing of the

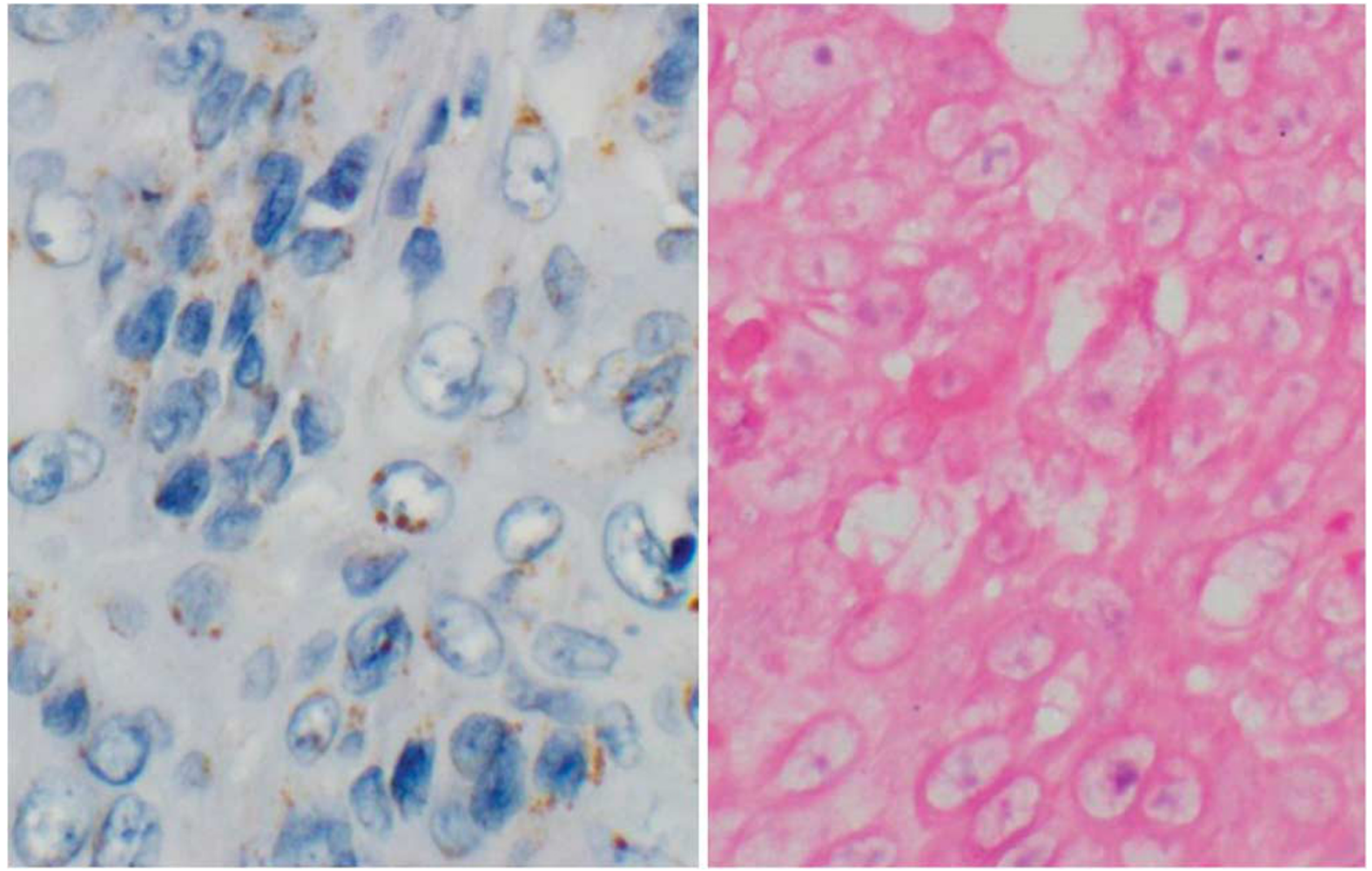

Figure 4 Dako in situ hybridization negative tumor showing nonspecific cytoplasmic and paranuclear signals (left panel), this same case had purple intranuclear signals and was interpreted as positive by Ventana positive (right panel), but was HPV negative by PCR. 
cervix. $^{14}$ However, this approach can be labor intensive and does not differentiate between integrated and episomal forms of the virus.

Molecular studies suggest that HPV-DNA in oropharyngeal tumors (overwhelmingly genotype 16) is present at high copy-numbers and is frequently integrated. ${ }^{33,34}$ Comparing HPV16 DNA and RNA detection by PCR, we observe a high concordance in DNA prevalence and E6/E7 expression, indicative of integration. However, the presence of E6/E7 RNA in DNA negative tumors, observed in two-thirds $(N=8 / 13)$ of discordant cases by PCR, might suggest the presence of integrated virus with loss or disruption of the $\mathrm{L} 1$ open reading frame. ${ }^{18,35}$ Following integration, the intact portion of the HPV16 genome can produce multiple E6 and E7 oncogene transcripts. ${ }^{18,36}$ This is of interest, as detection of HPV RNA in the tumor has recently been associated with better survival prediction than HPV DNA. ${ }^{8}$

Alternatively, the upregulation of p16 has been suggested as a marker of active HPV infection in head and neck squamous cell carcinoma, ${ }^{7}$ whereas inactivation of p16 by methylation is a common event in smoking associated cancers. ${ }^{37,38}$ p16 by immunohistochemistry is a method commonly used by pathologists, and its use with in situ hybridization for HPV DNA detection in a triage setting may help when HPV PCR analysis is not feasible., ${ }^{79-41}$ This combined testing may be of benefit, where cross-linking and DNA degradation induced by formalin fixation is a concern, as primer systems that target larger HPV-DNA fragments tend to have poorer detection rates in formalin-fixed and paraffin-embedded samples, compared with those targeting shorter DNA segments. ${ }^{42}$ For example, it has been suggested that the specificity of p16 immunohistochemistry in formalin-fixed and paraffin-embedded samples may be improved if followed by DNA analysis for p16 positive cases, when compared with E6 RT-PCR in fresh frozen tissue as the standard test. ${ }^{11}$ In our experience, p16 positive cases varied with respect to patterns of staining. However, relaxing the criteria for p16 positive immunohistochemistry tests did not improve performance in HPV16 E6/E7 RNA detection when followed by in situ hybridization or by MY-PCR.

We should note that despite using expert staining and evaluations, tests were performed on tissue microarrays, which do not reproduce the clinical diagnostic setting. This approach was chosen because of the high-throughput capability for conducting in situ hybridization and immunohistochemistry assays simultaneously on multiple samples. Although, multiple tissue microarray cores were selected by a pathologist for each tumor to reflect the diagnostic histopathology specimen, results may differ for clinical formalin-fixed and paraffin-embedded tissue sections.

Typically, HPV positive oropharyngeal squamous cell carcinomas were non-keratinizing with basa- loid-type tumor cells. We found that p16 immunohistochemistry continued to perform better than in situ hybridization, when compared with HPV detected by PCR in non-keratinizing squamous cell carcinomas and oropharyngeal tumors specifically. We also observed an inverse correlation between p16 expression and limited keratinization, which reflects non-keratinizing basaloid morphology.

In summary, although these results do not preclude the potential utility of assessing p16 expression as a biomarker for the clinical management of head and neck squamous cell carcinoma, ${ }^{28,43}$ particularly for oropharyngeal SCC, further analytical evaluation by blinded trials with multiple testing methods is warranted before these assays should be recommended for clinical HPV detection.

\section{Acknowledgements}

We thank the participants of this study; Catherine Sarta for her time and effort spent enrolling and following participants and with data entry; Gregory Rosenblatt for his assistance with data management; and Jaya Sunkara for her assistance with the immunohistochemistry protocol. This project is supported in part by NIH grant CA115243 (to NFS) and the Department of Pathology, Albert Einstein College of Medicine/Montefiore Medical Center.

\section{Disclosure/conflict of interest}

The authors declare no conflict of interest.

\section{References}

1 Jemal A, Siegel R, Ward E, et al. Cancer statistics, 2006. CA Cancer J Clin 2006;56:106-130.

2 Schlecht NF. Prognostic value of human papillomavirus in the survival of head and neck cancer patients: an overview of the evidence. Oncol Rep 2005;14: 1239-1247.

3 Ang KK, Harris J, Wheeler R, et al. Human papillomavirus and survival of patients with oropharyngeal cancer. N Engl J Med 2010;363:24-35.

4 Brandwein-Gensler M, Smith RV. Prognostic indicators in head and neck oncology including the new 7th edition of the AJCC staging system. Head Neck Pathol 2010;4:53-61.

5 Fakhry C, Gillison ML. Clinical implications of human papillomavirus in head and neck cancers. J Clin Oncol 2006;24:2606-2611.

6 Mulvany NJ, Allen DG, Wilson SM. Diagnostic utility of p16INK4a: a reappraisal of its use in cervical biopsies. Pathology 2008;40:335-344.

7 Smith EM, Wang D, Kim Y, et al. P16INK4a expression, human papillomavirus, and survival in head and neck cancer. Oral Oncol 2008;44:133-142.

8 Shi W, Kato H, Perez-Ordonez B, et al. Comparative prognostic value of HPV16 E6 mRNA compared with in situ hybridization for human oropharyngeal 
squamous carcinoma. J Clin Oncol 2009;27: 6213-6221.

9 Kong CS, Balzer BL, Troxell ML, et al. p16INK4A immunohistochemistry is superior to HPV in situ hybridization for the detection of high-risk HPV in atypical squamous metaplasia. Am J Surg Pathol 2007;31:33-43.

10 Holladay EB, Logan S, Arnold J, et al. A comparison of the clinical utility of p16(INK4a) immunolocalization with the presence of human papillomavirus by hybrid capture 2 for the detection of cervical dysplasia/ neoplasia. Cancer 2006;108:451-461.

11 Smeets SJ, Hesselink AT, Speel EJ, et al. A novel algorithm for reliable detection of human papillomavirus in paraffin embedded head and neck cancer specimen. Int J Cancer 2007;121:2465-2472.

12 Singhi AD, Westra WH. Comparison of human papillomavirus in situ hybridization and p16 immunohistochemistry in the detection of human papillomavirus-associated head and neck cancer based on a prospective clinical experience. Cancer 2010;116: 2166-2173.

13 Agoston ES, Robinson SJ, Mehra KK, et al. Polymerase chain reaction detection of HPV in squamous carcinoma of the oropharynx. Am J Clin Pathol 2010;134: 36-41.

14 Castle PE, Schiffman M, Gravitt PE, et al. Comparisons of HPV DNA detection by MY09/11 PCR methods. J Med Virol 2002;68:417-423.

15 Braakhuis BJ, Snijders PJ, Keune WJ, et al. Genetic patterns in head and neck cancers that contain or lack transcriptionally active human papillomavirus. J Natl Cancer Inst 2004;96:998-1006.

16 van Houten VM, Snijders PJ, van den Brekel MW, et al. Biological evidence that human papillomaviruses are etiologically involved in a subgroup of head and neck squamous cell carcinomas. Int J Cancer 2001;93: 232-235.

17 DeLong ER, DeLong DM, Clarke-Pearson DL. Comparing the areas under two or more correlated receiver operating curves: a nonparametric approach. Biometrics 1988;44:837-845.

18 zur Hausen H. Papillomaviruses and cancer: from basic studies to clinical application. Nat Rev Cancer 2002;2:342-350.

19 Fakhry C, Westra WH, Li S, et al. Improved survival of patients with human papillomavirus-positive head and neck squamous cell carcinoma in a prospective clinical trial. J Natl Cancer Inst 2008;100: 261-269.

20 Hallez S, Simon P, Maudoux F, et al. Phase I/II trial of immunogenicity of a human papillomavirus (HPV) type $16 \mathrm{E} 7$ protein-based vaccine in women with oncogenic HPV-positive cervical intraepithelial neoplasia. Cancer Immunol Immunother 2004;53: 642-650.

21 Wang XG, Revskaya E, Bryan RA, et al. Treating cancer as an infectious disease-viral antigens as novel targets for treatment and potential prevention of tumors of viral etiology. PLoS One 2007;2:e1114.

22 Allen CT, Lewis Jr JS, El-Mofty SK, et al. Human papillomavirus and oropharynx cancer: biology, detection and clinical implications. Laryngoscope 2010; 120:1756-1772.

23 Wittekindt C, Gultekin E, Weissenborn SJ, et al. Expression of p16 protein is associated with human papillomavirus status in tonsillar carcinomas and has implications on survival. Adv Otorhinolaryngol 2005;62:72-80.

24 El-Mofty SK, Patil S. Human papillomavirus (HPV)related oropharyngeal nonkeratinizing squamous cell carcinoma: characterization of a distinct phenotype. Oral Surg Oral Med Oral Pathol Oral Radiol Endod 2006;101:339-345.

25 Kim SH, Koo BS, Kang S, et al. HPV integration begins in the tonsillar crypt and leads to the alteration of p16, EGFR and c-myc during tumor formation. Int J Cancer 2007;120:1418-1425.

26 Charfi L, Jouffroy T, de Cremoux P, et al. Two types of squamous cell carcinoma of the palatine tonsil characterized by distinct etiology, molecular features and outcome. Cancer Lett 2008;260:72-78.

27 Kuo KT, Hsiao CH, Lin CH, et al. The biomarkers of human papillomavirus infection in tonsillar squamous cell carcinoma-molecular basis and predicting favorable outcome. Mod Pathol 2008;21:376-386.

28 Lewis Jr JS, Thorstad WL, Chernock RD, et al. p16 positive oropharyngeal squamous cell carcinoma:an entity with a favorable prognosis regardless of tumor HPV status. Am J Surg Pathol 2010;34: 1088-1096.

29 Unger ER. In situ diagnosis of human papillomaviruses. Clin Lab Med 2000;20:289-301, vi.

30 Shibata DK, Arnheim N, Martin WJ. Detection of human papilloma virus in paraffin-embedded tissue using the polymerase chain reaction. J Exp Med 1988;167:225-230.

31 Manos MM, Ting Y, Wright DK, et al. Use of polymerase chain reaction amplification for the detection of genital human papillomaviruses. Cancer Cells 1989;7:209-214.

32 Gravitt PE, Manos MM. Polymerase chain reactionbased methods for the detection of human papillomavirus DNA. IARC Sci Publ 1992;119:121-133.

33 Gillison ML, Koch WM, Shah KV. Human papillomavirus in head and neck squamous cell carcinoma: are some head and neck cancers a sexually transmitted disease? Curr Opin Oncol 1999;11:191-199.

34 Lindel K, Beer KT, Laissue J, et al. Human papillomavirus positive squamous cell carcinoma of the oropharynx: a radiosensitive subgroup of head and neck carcinoma. Cancer 2001;92:805-813.

35 Klaes R, Woerner SM, Ridder R, et al. Detection of high-risk cervical intraepithelial neoplasia and cervical cancer by amplification of transcripts derived from integrated papillomavirus oncogenes. Cancer Res 1999;59:6132-6136.

36 Chung $\mathrm{CH}$, Gillison ML. Human papillomavirus in head and neck cancer: its role in pathogenesis and clinical implications. Clin Cancer Res 2009;15: 6758-6762.

37 Soria JC, Rodriguez M, Liu DD, et al. Aberrant promoter methylation of multiple genes in bronchial brush samples from former cigarette smokers. Cancer Res 2002;62:351-355.

38 Toyooka S, Maruyama R, Toyooka KO, et al. Smoke exposure, histologic type and geographyrelated differences in the methylation profiles of nonsmall cell lung cancer. Int J Cancer 2003;103: 153-160.

39 Klingenberg B, Hafkamp HC, Haesevoets A, et al. p16 INK4A overexpression is frequently detected in tumour-free tonsil tissue without association with HPV. Histopathology 2010;56:957-967. 
40 Licitra L, Perrone F, Bossi P, et al. High-risk human papillomavirus affects prognosis in patients with surgically treated oropharyngeal squamous cell carcinoma. J Clin Oncol 2006;24:5630-5636.

41 Kumar B, Cordell KG, Lee JS, et al. EGFR, p16, HPV Titer, Bcl-xL and p53, sex, and smoking as indicators of response to therapy and survival in oropharyngeal cancer. J Clin Oncol 2008;26: 3128-3137.
42 Baay MF, Quint WG, Koudstaal J, et al. Comprehensive study of several general and type-specific primer pairs for detection of human papillomavirus DNA by PCR in paraffin-embedded cervical carcinomas. J Clin Microbiol 1996;34:745-747.

43 Lassen P, Eriksen JG, Hamilton-Dutoit S, et al. HPVassociated p16-expression and response to hypoxic modification of radiotherapy in head and neck cancer. Radiother Oncol 2010;94:30-35.

Supplementary Information accompanies the paper on Modern Pathology website (http://www.nature.com/ modpathol) 